\title{
Evaluation of Tensile Strength of Jute Fiber Reinforced Polypropylene Composite
}

\section{Tazdik Patwary Plateau}

Department of Materials and Metallurgical Engineering, Bangladesh University of Engineering and Technology, Dhaka, Bangladesh

\author{
Email address: \\ ptazdik@gmail.com
}

\section{To cite this article:}

Tazdik Patwary Plateau. Evaluation of Tensile Strength of Jute Fiber Reinforced Polypropylene Composite. Advances in Materials. Vol. 6, No. 6, 2017, pp. 149-153. doi: 10.11648/j.am.20170606.15

Received: October 9, 2017; Accepted: October 24, 2017; Published: November 24, 2017

\begin{abstract}
Natural fiber (such as flax, hemp, jute, kenaf, etc.) reinforced polymer resin composites are biodegradable composites as they are using fibers from renewable sources. It is also better than synthetic fibers (such as glass, carbon, ceramic fibers, etc.) in a sense of environmental cause. In this research work, jute fiber reinforced polypropylene matrix composites have been developed using hot compression molding technique. Various process parameters are used such as fiber condition (untreated and alkali treated), fiber sizes $(1,2$ and $4 \mathrm{~mm})$ and percentages $(5 \%, 10 \%$ and $15 \%$ by weight) in this research work. Tensile test and optical microscopy are used for characterization. Effect of tensile strength has a linear relation with fiber size and fiber percentage but after a certain size and percentage, the tensile strength shows an inverse relation with the fiber length and fiber percentage. There is no significant change has been observed for treated and untreated jute fiber reinforcement.
\end{abstract}

Keywords: Natural Fiber, Jute Fiber, Polypropylene, Composite, Tensile Strength

\section{Introduction}

Tensile strength varies material to material. Such as metals have high tensile strength on the other hand plastics and foams have low tensile strength. According to the properties of materials, a bubble chart is invented which is called material-property chart. But in this chart some empty spaces which are called voids. Reason of formation of this voids is the unavailability of materials having high tensile strength with low density and low tensile strength with high density. In case of looking forward to the innovation of newer material, composite material is developed. In composite materials, a matrix and a reinforced material is introduced. In this work, a composite of jute fiber reinforced polypropylene is developed and change of tensile strength is observed. Now all over the world, it is practiced to develop natural fiber reinforced plastic materials to have greater tensile properties. These natural fibers also reduced the total cost of materials. Moreover, jute fiber is available in Bangladesh; like this the reinforced material can be altered for different countries and different environments. There are other advantages of using

jute fiber for this work like as acceptable specific strength properties, low cost, low density, high toughness, good thermal properties, and so on. Low specific weight, which results in a higher specific strength and stiffness than glass is a benefit especially in parts designed for bending stiffness.

This is a thermoplastic composite and in case of this composite adhesion between jute fiber reinforcement and polypropylene composite is not so good. Because of this problem an alkali is used. Sodium hydroxide is used in this research work. Sodium hydroxide increases the interfacial bonding between fiber and matrix.

In this project, jute fiber reinforced polypropylene composites were prepared under various processing parameters using hot compression molding technique. The goal of this work is to understand the changes of tensile strength under various process parameters. Optical microscopy was done to show the conditions of fiber with increased fiber loading.

\section{Experimental}

\subsection{Material}

The composites were produced using treated (jute fibers were treated by Bangladesh Jute Research Institute, Dhaka, 
Bangladesh, with $20 \%$ sodium hydroxide) and untreated jute fiber and polypropylene pellets. The treated and untreated jute fibers were chopped into various lengths of 1, 2 and 4 $\mathrm{mm}$. For all lengths of fibers, composites were developed with 5, 10 and $15 \%$ (by weight) of jute.

\subsection{Composite Fabrication}

The chopped fibers were sieved with 1, 2 and $4 \mathrm{~mm}$ sieves for obtaining the desired variation in jute fiber length. The fibers were conditioned at $110^{\circ} \mathrm{C}$ for 24 hours to remove moisture and polypropylene was also conditioned at the same temperature. Proper proportion of fibers $(5,10$ and $15 \%$ by weight for each of 1,2 and $4 \mathrm{~mm}$ length) and polypropylene were then properly blended in the blender to get a homogeneous mixture for each length type. The mixture was placed in a mold and composites were made with $50 \mathrm{kN}$ load at $180^{\circ} \mathrm{C}$.

\subsection{Metallography}

Conventional metallography of selected specimens was done under metallurgical microscope.

\section{Results and Discussion}

Firstly, selected specimens were observed by the optical metallurgical microscope. Secondly, tensile testing specimens were prepared according to ASTM specification and were tested.

\subsection{Metallographic Observation}

The examination under metallurgical microscope shows the variation of fiber wt. percentages in different jute fiber composites (Figure 1). As evident from the figure, with the increase of fiber percentage in the composite, probability of entanglement of fiber increases. This is due to strong interhydrogen bonding between fibers.

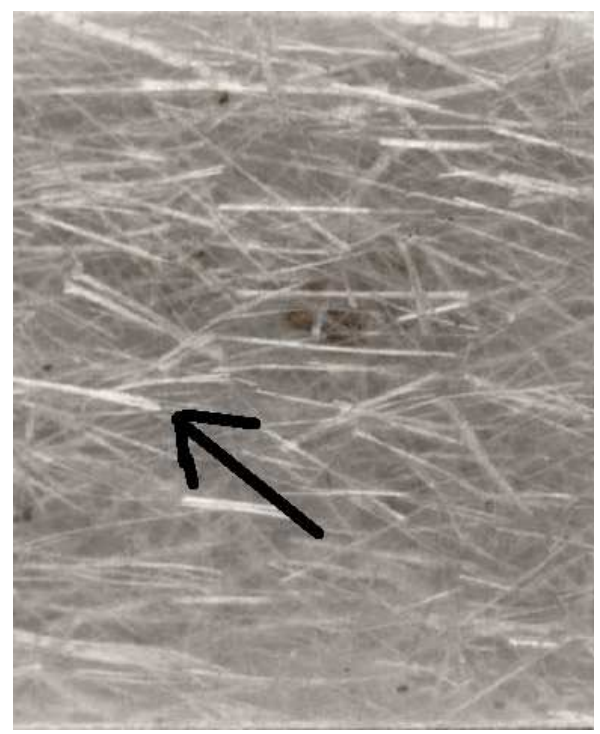

(a)

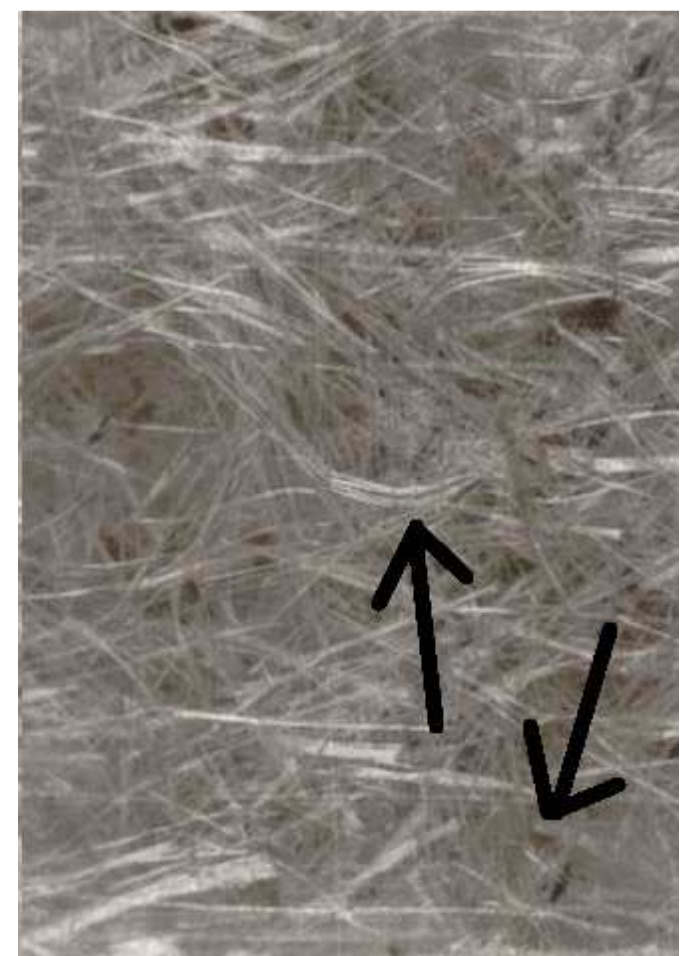

(b)

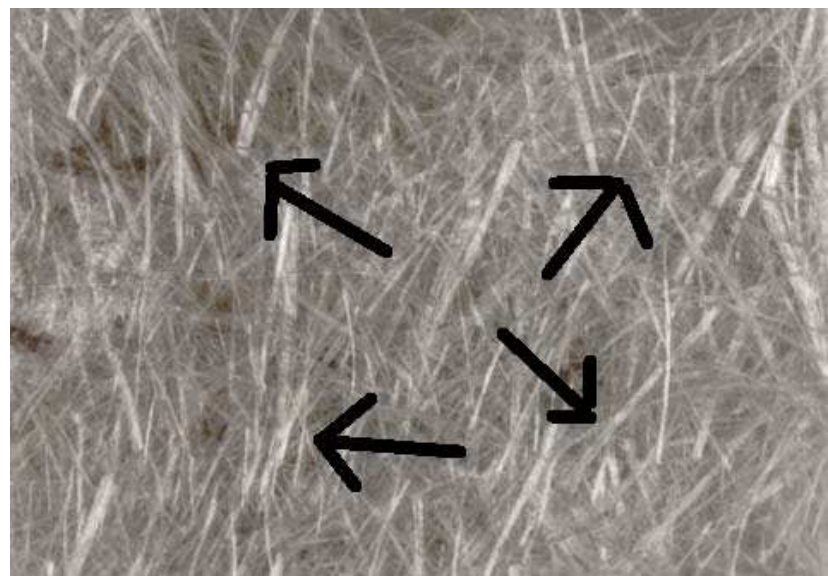

(c)

Figure 1. Optical microscopy of jute fiber reinforced polymer composites reinforced with various fiber wt. percentages (a) $5 \%$ Fiber (b) $10 \%$ Fiber (c) 15\% Fiber. Entanglement of fibers is shown by the arrow. It is clear that $15 \%$ fiber composite has highest fiber entanglement compared to 5 \& $10 \%$ fiber composites.

\subsection{Tensile Strength}

The typical load-stroke curve obtained from the tensile test is shown in Figure 2. From this curve, it can be predicted that the failure behavior of the jute fiber reinforced thermoplastic composite is brittle type.

The tensile test results have been plotted in Figures $3 \& 4$ as a function of fiber length. From these figures, it is clear that as the fiber length increases, the value of tensile strength increases and then decreases. This observation is true for almost all cases (with exception in the case of 5\% treated specimens). 


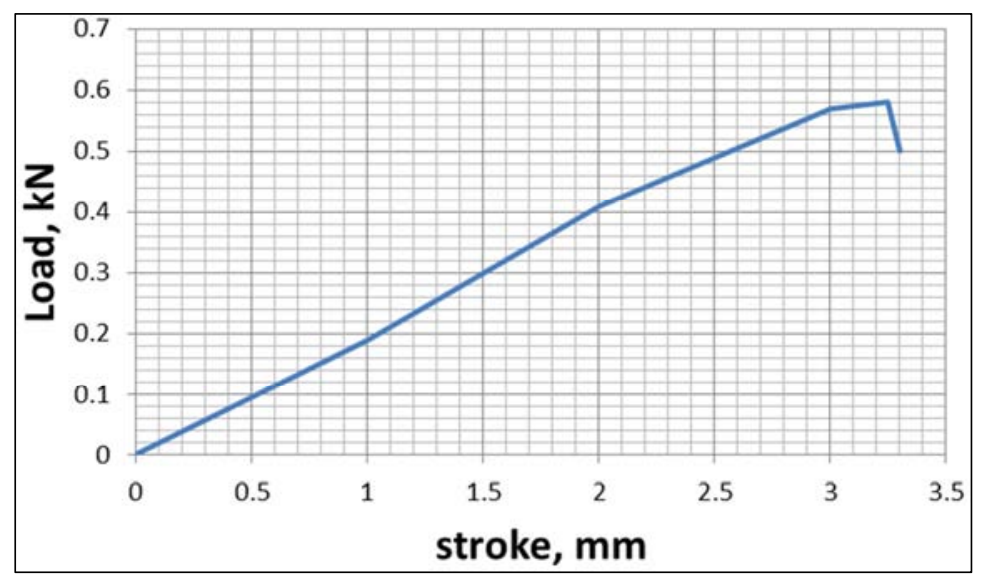

Figure 2. An observed load-stroke curve obtained from tensile test.

Figures $5 \& 6$ represent the relationship between fiber percentage $(\mathrm{wt} \%)$ and tensile strength value. As per these plots, in general, as the fiber percentage increases, the tensile strength also increases and then decreases.

As observed from the curves, tensile strength was increased to a maximum at $2 \mathrm{~mm}$ fiber length and then dropped. Also, tensile strength was found to increase to a maximum at $10 \%$ fiber (by weight) and then decreased. $2 \mathrm{~mm} \& 10 \%$ treated fiber composites gave better results than untreated fiber composites but not so distinguishable.
Fiber length has profound impact on the properties of composites. Besides holding the fibers together, the matrix has the important function of transferring applied load to the fibers. The efficiency of a fiber reinforced composite depends on the fiber-matrix interface and the ability to transfer stress from the matrix to the fiber. In small fiber size (here, $1 \mathrm{~mm}$ ), tensile strength is low due to the fact that length may be not sufficient enough for proper distribution of load. As proper length is not available for stress distribution, failure occurs easily.

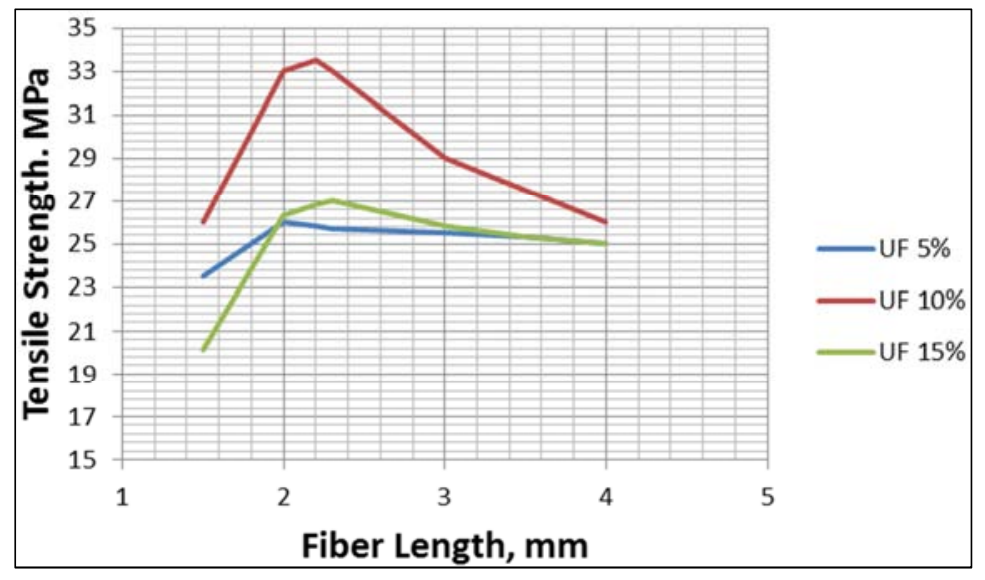

Figure 3. Tensile Strength of Untreated Jute Fiber Composites at Different Lengths.

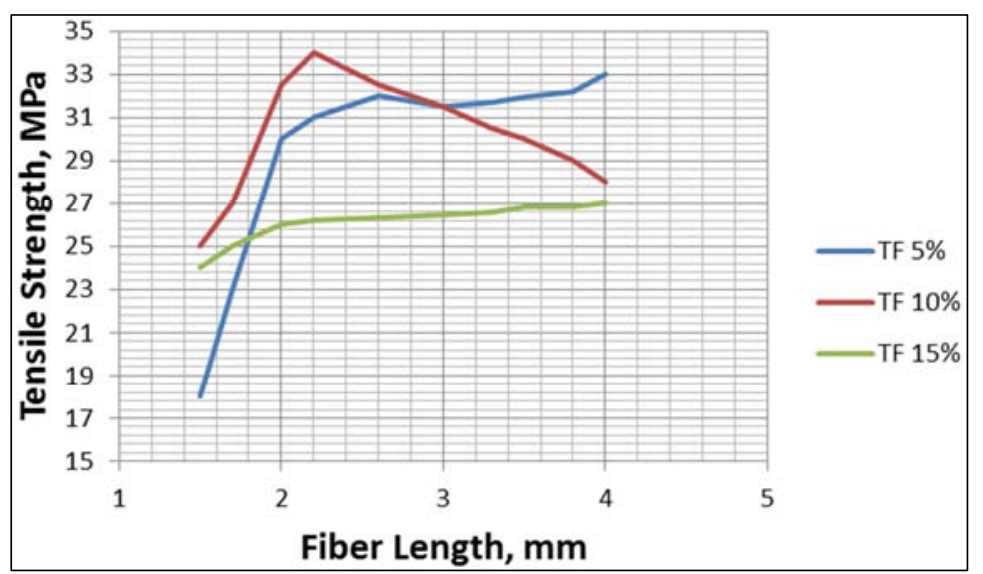

Figure 4. Tensile Strength of Treated Jute Fiber. 
Composites at Different Lengths

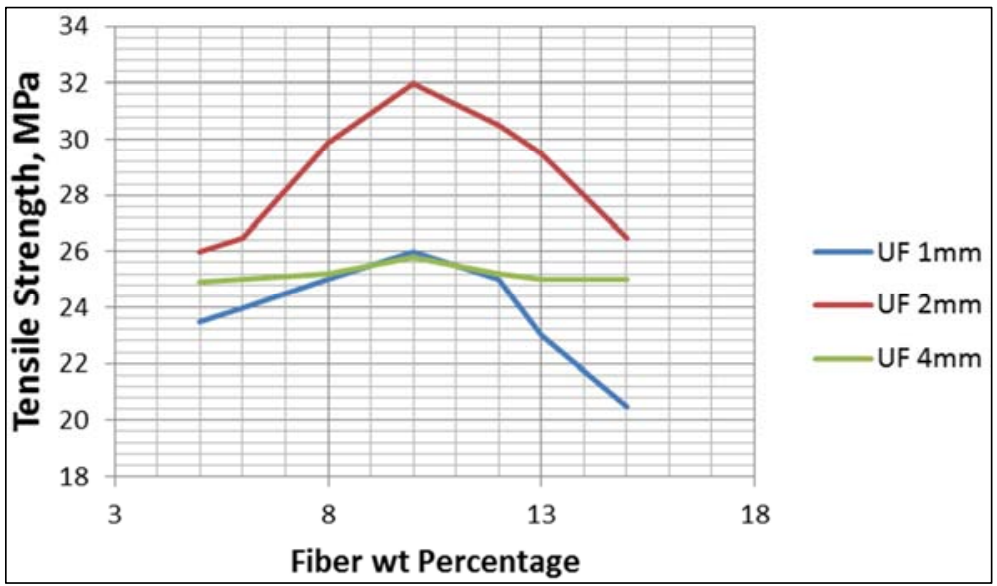

Figure 5. Tensile Strength of Untreated Jute Composites at Different Fiber wt. Percentages.

On the other hand, for the composites of longer fiber size (here, $4 \mathrm{~mm}$ ), tensile strengths were decreased compared to 2 $\mathrm{mm}$ fiber reinforced composites. The probable reason is that a long fiber may not become compatible with the matrix properly. Thus improper bonding occurs between the fibers and the matrix.

Moreover, fibers may be folded and there is no bonding between the folded and unfolded portion of fiber which resulted in a lower strength. Fiber entanglement may also contribute to reduce the strength.

For 5\% treated fiber composites, the exceptional behavior is probably that $4 \mathrm{~mm}$ size of fiber is still not enough to create fiber entanglement or folding inside the matrix.

In phenol formaldehyde/banana fiber composites, with the increase of fiber length tensile strength was found to be increased (Joseph et al., 2002). The trend of increase followed by decrease of tensile strength observed in current project was found in sisal/polypropylene composites.

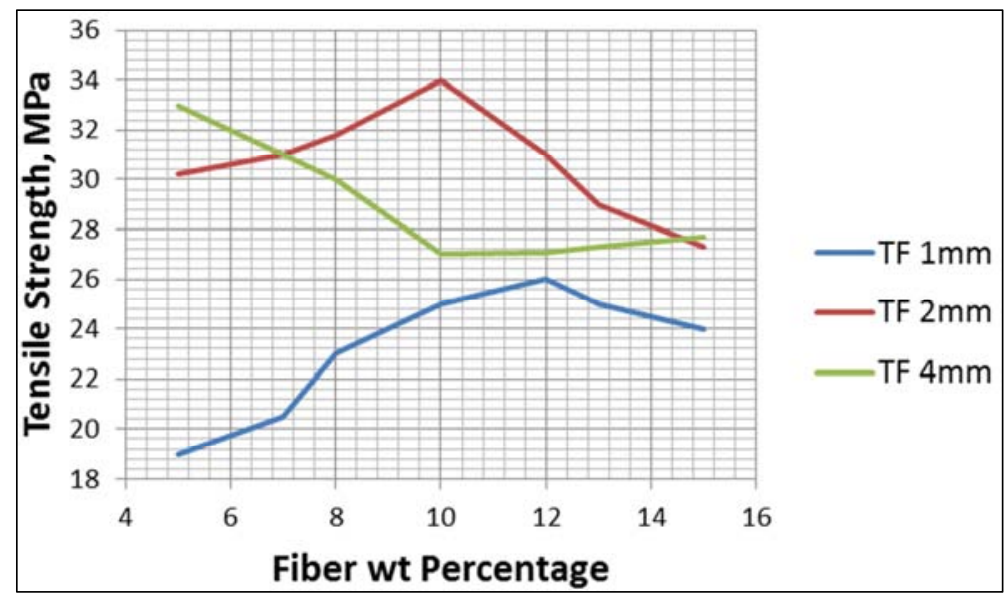

Figure 6. Tensile Strength of Treated Jute Fiber Composites atDifferent Fiber wt. Percentages.

According to Figures $5 \& 6$, after 10 wt. percent fiber as reinforcement in the composites, tensile strength was decreased with higher percentages of fiber. The incorporation of fibers into thermoplastics leads to poor dispersion of fibers due to strong inter fiber hydrogen bonding which holds the fibers together. Improper adhesion hinders the considerable increment of tensile strength. Thus, as fiber percentage increases, gathering of fibers takes place instead of dispersion and melted polypropylene cannot wet them properly due to non-entrance of melt through the adjacent two fibers. Since no adhesion is present between the fibers and fibers are also not bonded with matrix, failure occurs before attaining the theoretical strength of composite. Thus high fiber content was limited by the incompatibility issue unless coupling agent is used.

It has been reported that initially strength may decrease after a slight increase in strength and then at very high fiber content it may again increase. In polypropylene/wood composites, tensile strength was found to decrease after a certain percentage of fiber.

\section{Results}

The tensile strengths of the uncoupled composites have values in close range for all fiber percentage levels. Without coupling agent, fiber content and fiber length do not have 
significant effects on composite tensile properties. There exist incompatibilities between the different surface properties of the polar fibers and non-polar polypropylene. Due to presence of hydroxyl and other polar groups in various constituents of natural fiber, the moisture uptake is high for dry fibers.

A strong sodium hydroxide treatment may remove lignin, hemicellulose and other alkali soluble compounds from the surface of the fibers to increase the numbers of reactive hydroxyl groups on the fiber surface available for chemical bonding. So, strength should be higher than untreated fiber composites. The probable cause of this unlike phenomenon may be, alkali react on the cementing materials of the fiber specially hemicellulose which leads to the splitting of the fibers into finer filaments.

As a result, wetting of fiber as well as bonding of fiber with matrix may improve which consequently make the fiber more brittle. Under stress, these fibers break easily.

As evident from Figures 3-6, tensile strength was not significantly improved by alkali treatment. But, alkali treatment generally increases the strength of natural fiber composites.

As a coupling agent, MAPP may be used to enhance interfacial adhesion that may react or interact favorably with the hydroxyl group on the fiber surface. Use of coupling agent reduces the number of fiber pull-out.

Tensile Strength cannot take part in stress transfer mechanism. To improve affinity and adhesion between fiber and thermoplastic, chemical coupling agents can be used so that tensile strength increases.

So, high concentration of sodium hydroxide may increase the rate of hemicellulose dissolution which will finally lead to strength deterioration. Moreover, unnecessary extra time in treatment may also cause increment of hemicellulose dissolution.

\section{Conclusions}

From metallographic optical microscope, it is clearly showed that with the increase of fiber wt. percentage, entanglement of fibers occurs. In the case of fiber length, 2 $\mathrm{mm}$ jute fiber composites give better tensile strength over 1 $\& 4 \mathrm{~mm}$ jute fiber composites. In the case of fiber amount, 10 percent fiber (by weight) composites has better tensile strength compared to $5 \& 15 \mathrm{wt}$. percent fiber composites.

\section{Acknowledgements}

The author is very much grateful to Bangladesh University of Engineering and Technology (BUET) to provide necessary help for this research and also to the authority of BCSIR, Dhaka-1205, Bangladesh, for allowing them to use the laboratory facilities there. An unconditional thanks also goes to Bangladesh Jute Research Institute, Dhaka, Bangladesh, because of the treatment of jute fiber.

\section{References}

[1] Gassan, J. and Bledzki, A. K. (1997): Influence of FiberSurface Treatment on The Mechanical Properties of JutePolypropylene Composites, Composites - Part A: Applied Science and Manufacturing, Vol. 28, No. 12, pp. 1001-1005.

[2] Beg, M. D. H. and Pickering, K. L. (2004): Effect of Fiber Pretreatment on the Mechanical Properties of Wood/Polypropylene Composites, Proceedings of SPPM, 2527 February 2004, Dhaka, pp. 240-247.

[3] Karnani, R., Krishnan, M. and Narayan, R. (1997): BiofiberReinforced Polypropylene Composites, Polymer Engineering and Science, Vol. 37, No. 2, pp. 476-483.

[4] Mohanty, S., Nayak, S. K., Verma, S. K. and Tripathy, S. S. (2004): Effect of MAPP as a Coupling Agent on the Performance of Jute-PP Composites, Journal of Reinforced Plastics and Composites, Vol. 23, No. 6, pp. 625-637.

[5] Alam, S. N., Pickering, K. L. and Fernyhough, A. (2004): The Characterization of Natural Fibers \& Their Interfacial \& Composite Properties, Proceedings of SPPM, 25-27 February 2004, Dhaka, pp. 248-256.

[6] Wollerdorfer, M. and Bader, H. (1998): Influence of Natural Fibres on the Mechanical Properties of Biodegradable Polymers, Industrial Crops and Products, Vol. 8, No. pp. 105112.

[7] Joseph, S., Thomas, S., Sreekala, M. S., Oommen, Z. and Koshy, P. (2002): A Comparison of the Mechanical Properties of Phenol Formaldehyde Composites Reinforced With Banana Fibres And Glass Fibres, Composites Science and Technology, Vol. 62, No. 14, pp. 1857-1868.

[8] H. M. M. A. Rashed, M. A. Islam and F. B. Rizvi (2006); Effects of Process Parameters on Tensile Strength of Jute Fiber Reinforced Thermoplastic Composites, Journal of Naval Architecture and Marine Engineering 3(2006) pp 1-6.

[9] Tazdik Patwary Plateau, Md. Majidul Haque Bhuiyan (2017), A Heuristic Proposition of Efficient Copper-Electrodeposited p-type Thin Film for CZTS Solar Cell, Electrical, Computer and Communication Engineering (ECCE), International Conference(2017), DOI: 10.1109/ECACE.2017.7912887.

[10] William D. Callister, Jr., Materials Science and Engineering, An Introduction, Seventinth Edition. 